Journal of Antimicrobial Chemotherapy (1991) 27, Suppl. C, 21-28

\title{
Efficacy of once- and thrice-daily dosing of aminoglycosides in in-vitro models of infection
}

\author{
Jürg Blaser \\ Departement für Innere Medizin, Universitätsspital, CH-8091 Zürich, Switzerland
}

\begin{abstract}
The bactericidal efficacy of amikacin, isepamicin and netilmicin was studied against Pseudomonas aeruginosa and Serratia marcescens over a treatment period of $30 \mathrm{~h}$ using two one-compartment in-vitro models with differently designed culture compartments. High bacterial inocula were exposed to fluctuating drug concentrations, simulating human serum concentrations $\left(t_{1 / 2}=2 \mathrm{~h}\right)$ during clinical treatment. The same daily dose was administered as $1 \mathrm{~h}$ infusions given every $8 \mathrm{~h}$ or every $24 \mathrm{~h}$, resulting in peak concentrations of 8 and $24 \mathrm{mg} / 1$ for netilmicin, and 24 and $72 \mathrm{mg} / \mathrm{l}$ for amikacin and isepamicin, respectively. Once-daily dosing was more bactericidal during initial treatment in the in-vitro models $(P<0.01)$ and at least as effective as thrice-daily dosing in preventing bacterial regrowth, despite a prolonged period of subinhibitory drug concentration before administration of the second dose. Lower ratios of peak concentration to MIC were needed to achieve bactericidal activity ( $>99.9 \%$ reduction of cfu) after $24 \mathrm{~h}$ treatment against $S$. marcescens compared with $P$. aeruginosa $(P<0.01)$. All nine regimens providing peaks of at least four times the MIC were bactericidal against $S$. marcescens after $24 \mathrm{~h}$ exposure. In contrast, a bactericidal effect against $P$. aeruginosa occurred only during two of six experiments with peaks of four to nine times the MIC. Similar results were obtained in both in-vitro models of infection. These data suggest insufficient intrinsic activity of the aminoglycosides studied for single drug treatment of $P$. aeruginosa in the absence of host-defence mechanisms.
\end{abstract}

\section{Introduction}

Multiple daily dosing regimens of aminoglycosides have been used for more than three decades to treat systemic bacterial infections. However, data from in-vitro and experimental in-vivo studies suggest that once-daily dosing of aminoglycoside might be at least as efficacious and potentially less toxic (Frame et al., 1977; Blaser, Stone \& Zinner, 1985a; Gerber et al., 1989; Mattie, Craig \& Pechère, 1989). Single-daily dosing of aminoglycosides has also been studied clinically (Powell et al., 1983; Hollender et al., 1989). However, a large number of patients need to be evaluated in comparative studies to detect clinically significant differences in either efficacy or toxicity.

Optimal dosing of antibiotics has been studied in various in-vitro models of infection (Blaser \& Zinner, 1987). In the present study cultures of Pseudomonas aeruginosa and Serratia marcescens were treated with amikacin, netilmicin or isepamicin, simulating human serum pharmacokinetics. Each drug was administered either once- or thrice-daily.

21

(C) 1991 The British Society for Antimicrobial Chemotherapy 


\section{Methods}

Antibacterial efficacy of isepamicin, amikacin and netilmicin was studied in two in-vitro models of infection over treatment periods of $30 \mathrm{~h}$. Figures 1 and 2 show schematic drawings of the two one-compartment models ( $V$ and $G$ ), each with a differently designed growth chamber. In model $V$ the culture chamber consisted of a $10 \mathrm{ml}$ Vacutainer glass tube with siliconized glass walls. The cultures were not stirred and direct contact with air was maintained. In model $G$ bacteria were cultured in a $20 \mathrm{ml}$ glass and stainless steel compartment. The cultures were continuously stirred with a magnetic stirrer. The culture medium was not in direct contact with air and only the oxygen dissolved within the culture medium reservoir was pumped into the culture chamber.

Cultures of three strains of $P$. aeruginosa (one laboratory reference strain, ATCC 27853 , and two clinical isolates) were studied in both one-compartment models. In addition, three clinical isolates of $S$. marcescens were exposed to the antibiotics in model G. High bacterial inocula of $5 \times 10 \% \mathrm{ml}$ in a volume of $10 \mathrm{ml}$ were exposed to fluctuating drug concentrations, simulating human serum concentrations during treatment. The elimination half-lives were defined according to the ratio of flow rate to culture volume and adjusted to $2 \mathrm{~h}$ (Blaser \& Zinner, 1987). For each drug the same daily dose was administered as 1-h infusions given either every $24 \mathrm{~h}$ (q24 h) or every $8 \mathrm{~h}(\mathrm{q} 8 \mathrm{~h})$. Amikacin and isepamicin doses were three times higher than netilmicin for these experiments to account for the differences in dosing during clinical therapy. Concentrations were measured with fluorescence polarization immunoassay $\left(\mathrm{TD}_{\mathbf{x}}\right.$, Abbott). Peak concentrations for the $\mathrm{q} 24 \mathrm{~h}$ and $\mathrm{q} 8 \mathrm{~h}$ regimens were 24 and $8 \mathrm{mg} / \mathrm{l}$ for netilmicin, and 72 and $24 \mathrm{mg} / \mathrm{l}$ for amikacin and isepamicin, respectively. For both amikacin regimens measured and predicted concentrations are shown in Figure 3(a).

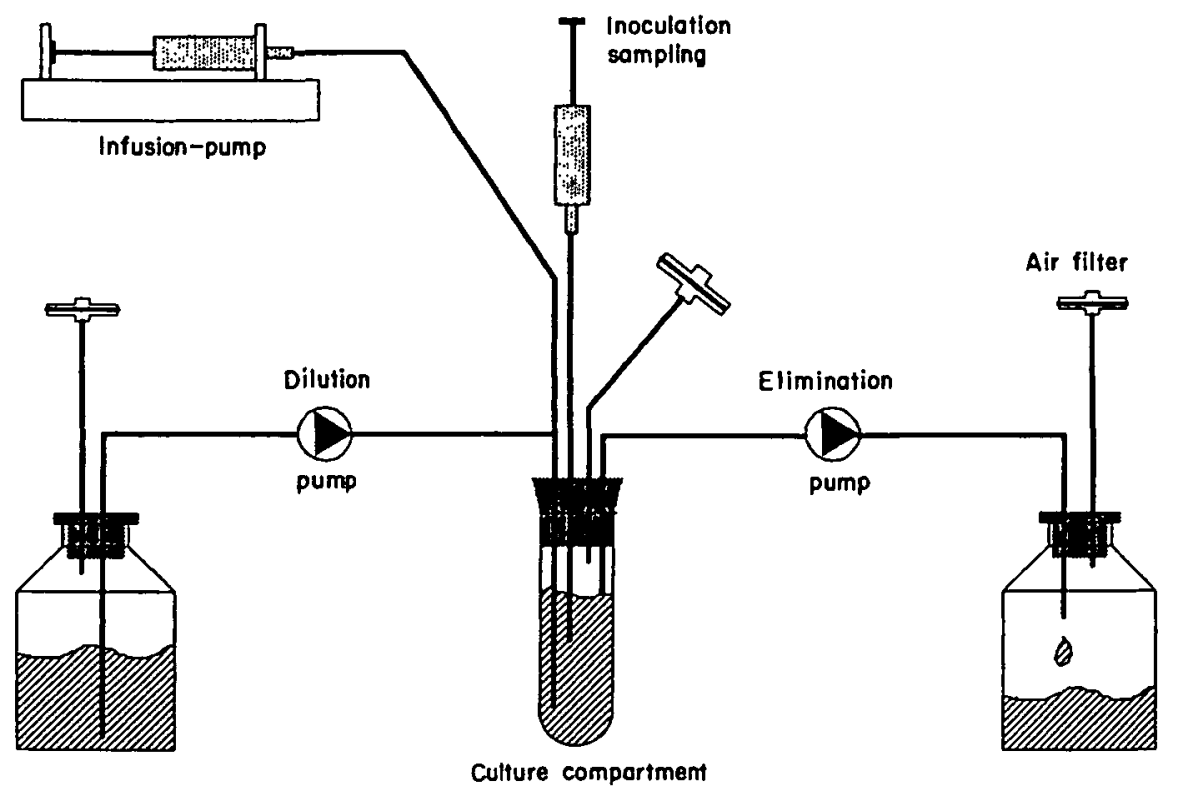

Figure 1. Schematic drawing of a one-compartment model with a $10 \mathrm{ml}$ Vacutainer tube as the growth chamber (model V). The glass walls of the tube were treated with silicon. The culture medium was not stirred and was in direct contact with air. 

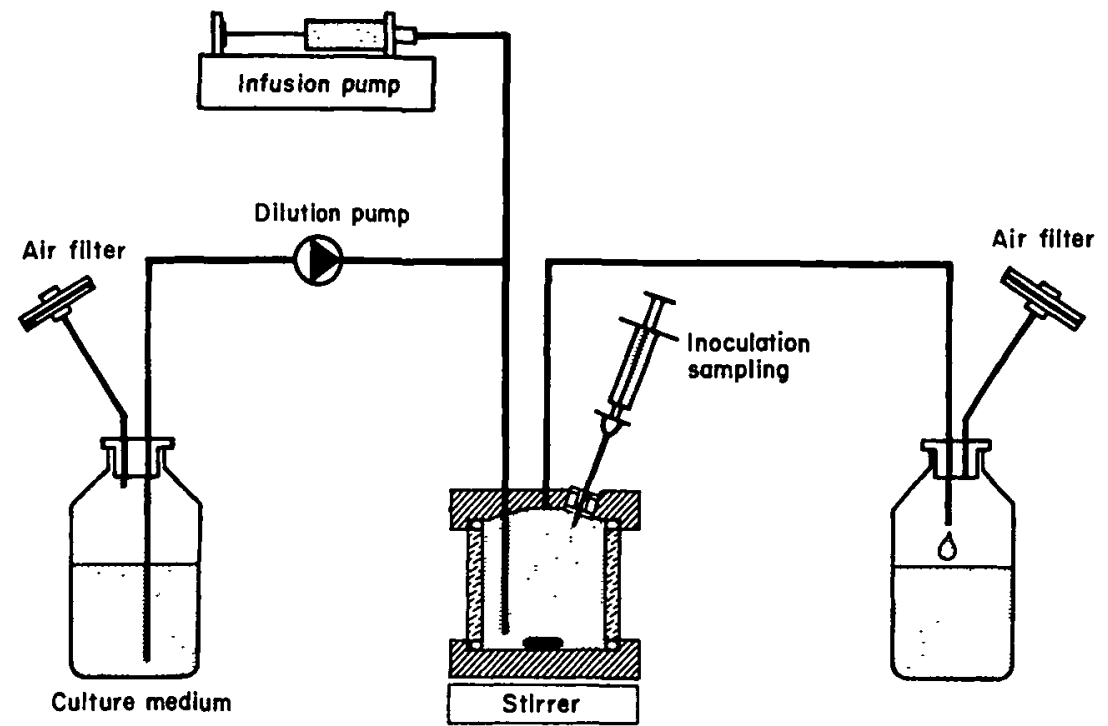

Figure 2. Schematic drawing of a one-compartment model with a $20 \mathrm{ml}$ glass and stainless steel culture compartment (model G). The model excludes direct air contact within the culture compartment. The culture is stirred continuously by a magnetic stirrer.
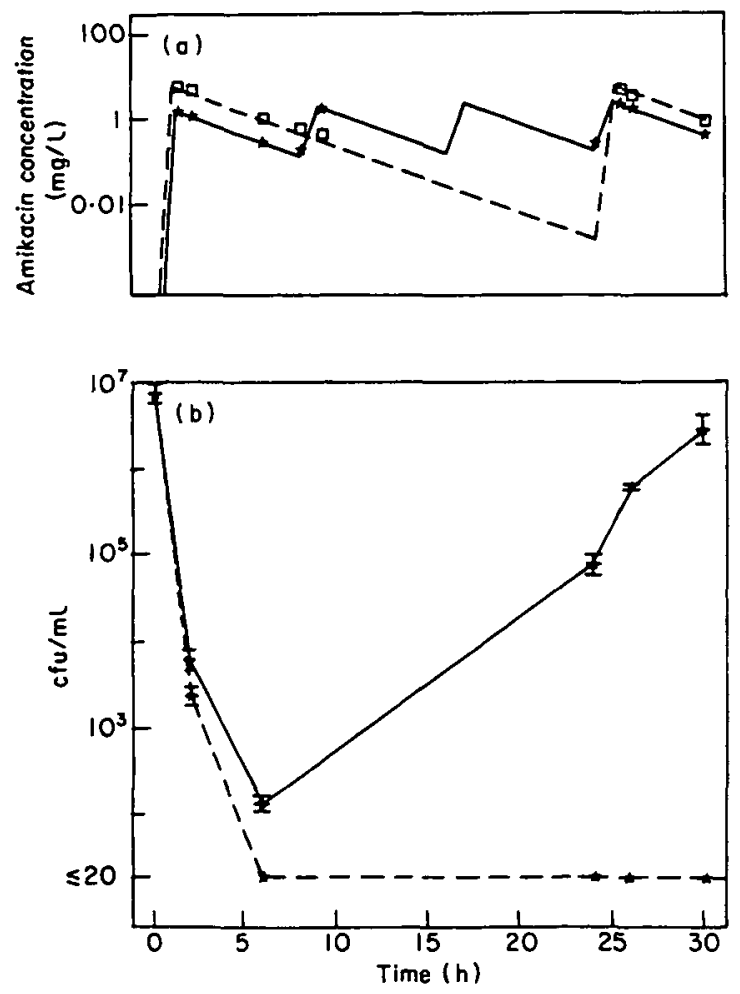

Figure 3. Impact of dosage regimen of amikacin against $P$. aeruginosa 27853 in a one-compartment model (model G). (a) Measured (q24 h, $\square ; q_{8} \mathrm{~h},{ }^{*}$ ) and predicted (q24 h, - -; q8 h, - drug concentrations in the culture chamber. (b) The effect of both regimens $(q 24 \mathrm{~h},---; q 8 \mathrm{~h}, \longrightarrow$ ) on the number of cfus (geometric mean and range of duplicate experiments). 
Susceptibility to aminoglycosides was determined by a tube-dilution method in $2 \mathrm{ml}$ volumes containing inocula of $5 \times 10^{6} \mathrm{cfu} / \mathrm{ml}$. MICs are listed in Table I. MBCs were two- to fourfold higher than MICs for the strains of pseudomonas, and identical to the MICs or twofold higher for the strains of serratia.

\section{Results}

All three aminoglycosides were rapidly and profoundly bactericidal in the in-vitro models against sensitive and intermediately sensitive strains of $P$. aeruginosa and $S$. marcescens (Tables I, II and Figure 3). Better initial bacterial killing was observed with q24 h regimens compared to q8 h regimens $(P<0.01$, Wilcoxon signed rank test). For example, against $P$. aeruginosa ATCC 27853 the $q 24 \mathrm{~h}$ and $\mathrm{q} 8 \mathrm{~h}$ regimens reduced the inocula after $6 \mathrm{~h}$ by $>5.3$ and $4.7 \log _{10}$ for amikacin, $>5.3$ and $3.6 \log _{10}$ for isepamicin, and 3 and $1.8 \log _{10}$ for netilmicin, respectively (means of duplicates; Model G). However, regrowth occurred within $24 \mathrm{~h}$ in all experiments except for the $\mathrm{q} 24 \mathrm{~h}$ regimens with amikacin. These experiments were repeated in a model with a differently designed growth chamber (model V; Figure 1). All three aminoglycosides showed similar dose response in this model (Table II). The bactericidal effect was slightly reduced (on an average by $0.8 \log _{10}$ ) and all cultures regrew within $30 \mathrm{~h}$.

Better bactericidal activity was observed with amikacin and isepamicin than with netilimicin (Table I). This relates to the lower ratios of peak concentration to MIC achieved with netilmicin. The MICs of the organisms studied were similar for all three drugs, but three times lower doses of netilmicin were administered, to account for differences in clinical dosing of these antibiotics.

Lower ratios of peak concentration to MIC were needed to achieve bactericidal activity ( $>99.9 \%$ fold reduction of cfu) after $24 \mathrm{~h}$ of treatment against $S$. marcescens compared to $P$. aeruginosa $(P=0.011$, Fisher's exact test, Table I). For $S$. marcescens a peak/MIC ratio of $\geqslant 4$ occurred for nine of the 18 combinations of strain, drug and dosing regimen and all of these nine combinations resulted in $\geqslant 99.9 \%$ reduction in viable bacteria. In contrast, for $P$. aeruginosa a peak/MIC ratio of $\geqslant 4$ only occurred for six of the 18 combinations of strain, drug and dosing regimen. More importantly, $99.9 \%$ reduction in bacterial numbers only occurred in two out of these six combinations. For example, despite identical ratios of amikacin peak concentration to MIC, lower activity was found in the kinetic model against $P$. aeruginosa 14974 compared to $S$. marcescens 77 . Similarly, isepamicin was less active against $P$. aeruginosa A-10 than against $S$. marcescens 76 , despite identical peak to MIC ratios. Netilmicin also was less active against $P$. aeruginosa 14974 than against $S$. marcescens 77 during exposure to identical peak to MIC ratios. No bactericidal effect was observed after $24 \mathrm{~h}$ with isepamicin against two strains of $P$. aeruginosa despite peaks of nine times the MIC. However, isepamicin was bactericidal against serratia even at a peak to MIC ratio of only 1.5 .

Although better initial bacterial killing was observed with $\mathrm{q} 24 \mathrm{~h}$ regimens compared to $\mathrm{q} 8 \mathrm{~h}$ regimens, comparable antibacterial efficacy was observed after $24 \mathrm{~h}$ during most aminoglycoside treatments with both dosing regimens (Tables I, II). However, for $P$. aeruginosa ATCC 27853 the $\mathrm{q} 24 \mathrm{~h}$ regimen resulted in more rapid and complete killing during the first $6 \mathrm{~h}$ of exposure (Figure 2). Moreover, there was no regrowth despite the prolonged period with subinhibitory concentrations before the second dose, whereas regrowth occurred during thrice-daily dosing (Figure 3, Table II). Similar results were obtained with $P$. aeruginosa A-10 (Table I). 


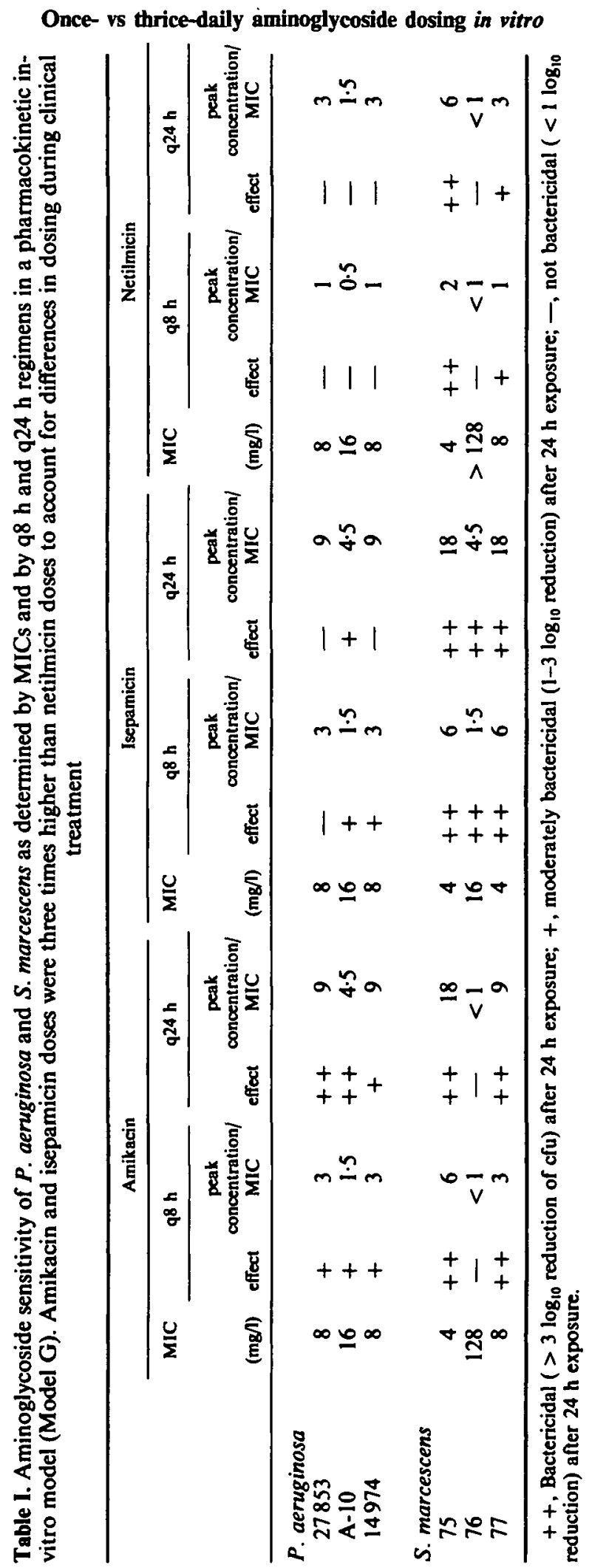


Table II. Bactericidal effects of six aminoglycoside treatment regimens after 6 and $24 \mathrm{~h}$ against $P$. aeruginosa (ATCC 27853). Inocula of $2-8 \times 10^{6} \mathrm{cfu} / \mathrm{ml}$ were treated in two pharmacodynamic models (Figures 1 and 2). Results are shown as cfu/ml 6 and $24 \mathrm{~h}$ after the start of treatment

\begin{tabular}{|c|c|c|c|c|}
\hline \multirow{2}{*}{$\begin{array}{l}\text { Drug and } \\
\text { timing of } \\
\text { bactericidal } \\
\text { effect }\end{array}$} & \multicolumn{2}{|c|}{ q8 h regimen } & \multicolumn{2}{|c|}{ q24 h regimen } \\
\hline & model $\mathrm{V}$ & model $\mathbf{G}$ & model V & model G \\
\hline $\begin{array}{c}\text { Amikacin } \\
6 \mathrm{~h} \\
24 \mathrm{~h}\end{array}$ & $\begin{array}{l}1 \times 10^{3} \\
5 \times 10^{6}\end{array}$ & $\begin{array}{l}1 \times 10^{2} \\
8 \times 10^{4}\end{array}$ & $\begin{array}{l}9 \times 10^{1} \\
1 \times 10^{7}\end{array}$ & $\begin{array}{r}2 \times 10^{1} \\
<2 \times 10^{1}\end{array}$ \\
\hline $\begin{array}{c}\text { Isepamicin } \\
6 \mathrm{~h} \\
24 \mathrm{~h} \\
\text { Netilmicin }\end{array}$ & $\begin{array}{l}2 \times 10^{3} \\
6 \times 10^{6}\end{array}$ & $\begin{array}{l}1 \times 10^{3} \\
2 \times 10^{7}\end{array}$ & $\begin{array}{l}8 \times 10^{1} \\
6 \times 10^{6}\end{array}$ & $\begin{array}{l}4 \times 10^{2} \\
8 \times 10^{6}\end{array}$ \\
\hline $\begin{array}{c}6 \mathrm{~h} \\
24 \mathrm{~h}\end{array}$ & $\begin{array}{l}5 \times 10^{5} \\
3 \times 10^{7}\end{array}$ & $\begin{array}{l}1 \times 10^{5} \\
7 \times 10^{6}\end{array}$ & $\begin{array}{l}9 \times 10^{2} \\
3 \times 10^{7}\end{array}$ & $\begin{array}{l}7 \times 10^{2} \\
3 \times 10^{7}\end{array}$ \\
\hline
\end{tabular}

\section{Discussion}

High peak to MIC ratios were required in both one-compartment in-vitro models to achieve and maintain a bactericidal effect against $P$. aeruginosa over a treatment period of $24 \mathrm{~h}$ or more. Similar results have been obtained previously in studies performed in a capillary two-compartment model (Blaser et al., 1987). These data suggest insufficient intrinsic activity of aminoglycosides for single drug treatment of high inocula of $P$. aeruginosa in the absence of any host-defence mechanisms. In contrast, relatively low peak to MIC ratios were sufficient to achieve and maintain a bactericidal effect during in-vitro treatment of serratia.

In-vitro pharmacokinetic models simulate the treatment of infection in the absence of host-defence mechanisms. During treatment of either Gram-negative or Gram-positive bacteria resistant subpopulations emerge unless the peak to MIC ratio is high enough to drastically reduce the bacterial inoculum within a few hours (Blaser $e t$ al., 1985a). Similarly, rapid emergence of resistant subpopulations has been reported during aminoglycoside treatment in neutropenic animals. Although the virulence and clinical relevance of the relatively slow growing resistant subpopulations has been documented both in animal and clinical studies (Gerber \& Craig, 1982; Olson et al., 1985), clinical experience suggests that this problem is more common in vitro than during treatment of patients, particularly of non-neutropenic patients. This discrepancy might relate to data suggesting enhanced activity of leucocytes against resistant pathogens selected during antibiotic exposure (Schlaeffer et al., 1990). However, treatment of infection in immunocompromised patients may still require antibiotic suppression of regrowth of aminoglycoside-resistant subpopulations. Combination therapy of an aminoglycoside plus a $\beta$-lactam antibiotic has been frequently used in this situation (Hilf et al., 1989). Data obtained with in-vitro models of infection suggest synergistic interactions due to prevention of the emergence of resistant subpopulations by $\beta$-lactams (Blaser et al., 1985b).

Once-daily dosing was more bactericidal during the first $6 \mathrm{~h}$ of drug exposure and at least as effective as thrice-daily dosing in preventing bacterial regrowth. The regimen of 
q24 $\mathrm{h}$ provides maximum peaks of eight times the MIC but the concentration falls below the MIC within only $6 \mathrm{~h}$ (equivalent to three half-lives). Therefore subinhibitory concentrations prevail during more than two-thirds of the dosing interval. These observations suggest either complete sterilization of the system or a prolonged postantibiotic effect in the presence of subinhibitory concentrations before administration of the next dose.

Data obtained in vitro support the concept of once-daily dosing of aminoglycosides and suggest further clinical evaluations. Current clinical trials of optimal dosing of aminoglycosides should provide information on the presence or absence of major differenes in efficacy or toxicity. However, for statistical reasons large numbers of patients will have to be enrolled in comparative studies to document differences in cure rates. Ethical, practical and statistical limitations of clinical studies, emphasize the importance of laboratory investigations in defining optimal dosing regimens.

\section{Acknowledgement}

I thank Alfred Stätzler for his excellent technical assistance.

\section{References}

Blaser, J., Stone, B. B. \& Zinner, S. H. (1985a). Efficacy of intermittent versus continuous administration of netilmicin in a new two compartment in-vitro model. Antimicrobial Agents and Chemotherapy 27, 343-9.

Blaser, J., Stone, B. B., Groner, M. C. \& Zinner, S. H. (1985b). Impact of netilmicin regimens on the activity of ceftazidime netilmicin combinations against Pseudomonas aeruginosa in an invitro pharmacokinetic model. Antimicrobial Agents and Chemotherapy 28, 64-8.

Blaser, J., Stone, B. B., Groner, M. C. \& Zinner, S. H. (1987). Comparative study with enoxacin and netilmicin in a pharmacodynamic model to determine importance of ratio of antibiotic peak concentration to MIC for bactericidal activity and emergence of resistance. Antimicrobial Agents and Chemotherapy 31, 1054-60.

Blaser, J. \& Zinner, S. H. (1987). In vitro models for the study of antibiotic activities. In Progress in Drug Research, Vol. 31 (Jucker, E. \& Meher, U., Eds), pp. 349-81. Birkhäuser Verlag.

Frame, P. T., Phair, J. P., Watanakunakorn, C. \& Bannister, T. W. P. (1977). Pharmacologic factors associated with gentamicin nephrotoxicity in rabbits. Journal of Infectious Diseases 135, 952-7.

Gerber, A. U. \& Craig, W. A. (1982). Aminoglycoside selected subpopulations of P. aeruginosa. Characterisation and virulence in normal and leukopenic mice. Journal of Laboratory and Clinical Medicine 100, 671-81.

Gerber, A. U., Kozak, S., Segessenmann, C., Flückiger, U., Bangerter, T. \& Greter, U. (1989). Once-daily versus thrice-daily administration of netilmicin in combination therapy of Pseudomonas aeruginosa infection in a man-adapted neutropenic animal model. European Journal of Clinical Microbiology and Infectious Diseases 8, 233-7.

Hilf, M. S., Yu, V. L., Sharp, J. A., Zuravleff, J. J., Korvick, J. A. \& Muder, R. R. (1989). Antibiotic therapy for Pseudomonas aeruginosa bacteremia: outcome correlations in a prospective study of 200 patients. American Journal of Medicine 87, 540-6.

Hollender, L. F., Bahnini, J., De Manzini, N., Lau, W. Y., Fan, S. T., Hermansyur, K. et al. (1989). A multicentre study of netilmicin once daily versus thrice daily in patients with appendicitis and other intra-abdominal infections. Journal of Antimicrobial Chemotherapy 23, 773-84.

Mattie, H., Craig, W. A. \& Pechère, J. C. (1989). Determinants of efficacy and toxicity of aminoglycosides. Journal of Antimicrobial Chemotherapy 24, 281-93.

Olson, B., Weinstein, R. A., Nathan, C. Chamberlin, W. \& Kabins, S. A. (1985). Occult aminoglycoside resistance in Pseudomonas aeruginosa epidemiology and implications for therapy and control. Journal of Infectious Diseases 152, 769-74. 
Powell, S. H., Thompson, W. L., Luthe, M. A., Stern, R. C., Grossniklaus, D. A., Bloxham, D. D. et al. (1983). Once-daily vs. continuous aminoglycoside dosing: efficacy and toxicity in animal and clinical studies of gentamicin, netilmicin, and tobramicin. Journal of Infectious Diseases 147, 918-32.

Schlaeffer, F., Blaser, J., Laxon, J. \& Zinner, S. (1990). Enhancement of leucocyte killing of resistant bacteria selected during exposure to aminoglycosides or quinolones. Journal of Antimicrobial Chemotherapy 25, 941-8. 